\title{
Tracheal resection with left lung anastomosis following right pneumonectomy
}

\author{
D. T . T H O M P S O N \\ From Baguley Hospital, Wythenshawe, Manchester 23
}

In a review of the literature on tracheal surgery no reference to an operation of this type could be found. Barclay, McSwan, and Welsh (1957) reported a case of resection of the lower end of the trachea with anastomosis of the right main bronchus to the trachea and implantation of the left main bronchus into the right main bronchus, i.e., two lungs were available for inflation during the operation.

The problems involved in undertaking an anastomosis of a single lung were threefold: (1) to obtain adequate gaseous exchange during anaesthesia while anastomosis of the severed but remaining lung was being carried out; (2) to achieve an 'airtight' anastomosis - any leak of air would endanger life by a pneumothorax on the side of the remaining lung; and (3) to ensure and maintain complete expansion of the anastomosed lung until oedema and inflammation had subsided and healing had occurred.

\section{CASE REPORT}

In early September 1965 the patient, a 52-year-old, well-built man, first experienced a fairly sudden sharp stabbing pain, aggravated by exertion, in the right inframammary region.

A week after the onset of symptoms he developed the clinical features of pneumonia on the right side, and was admitted to hospital.

He developed a cough productive of blood-stained sputum, but this cleared after treatment.

Serial radiographs showed consolidation-collapse of the right lower lobe, and bronchoscopy a short while later revealed a constricting tumour obstructing the bronchus at the level of the right middle lobe bronchial orifice. A specimen taken for microscopy confirmed the neoplastic nature of the constriction, it being poorly differentiated, squamous-cell in type.

There were no bronchoscopic contraindications to surgery, so the patient was transferred to a surgical ward. Respiratory function tests indicated that he could tolerate a pneumonectomy.
In October he was submitted to a right thoracotomy through the undivided sixth rib bed, and at this operation a tumour within the right lower lobe was found spreading medially to involve the pericardium.

A pneumonectomy was considered technically feasible, but to secure and deal with pulmonary veins intrapericardial clamping of the left atrium was necessary.

The pulmonary artery was clamped, transfixed, and $\vec{\emptyset}$ divided, and its cut end was oversewn.

Better access to the right main bronchus was thus obtained, and a mass of neoplastically involved glands was seen extending to the carina, and carcinomatous tissue was invading the origin of the left main bronchus and the carina (Fig. 1).

The tumour, thus situated, rendered impossible safe $\stackrel{\unrhd}{\varnothing}$ closure by usual techniques through healthy tissue $\overrightarrow{\vec{A}}$ of the right main bronchus following removal of the $\frac{\text { 일 }}{3}$ lung. It was decided to proceed to resection of the lower end of the trachea with the carina and the origin of the left main bronchus.

TECHNIQUE The right main bronchus was divided and the mobilized lung and mediastinal glands were $x$ removed.

The double-lumen Carlen's tube was removed and a single-lumen endotracheal tube was passed into theo left main bronchus and impacted in its lumen.

The carina and left main bronchus were theno incised as shown in Fig. 2, and this segment was removed as a 'jacket' from around the intrabronchialo tube.

A triangular wedge of cartilaginous portion of the trachea was then excised, the amount taken being judged to allow accurate end-to-end anastomosis between the trachea and the left main bronchus afterw reconstruction of the tracheal lumen (Fig. 3).

By impacting the endotracheal tube into the lefto main bronchus, inflating for one minute, then withdrawing the tube into the trachea for one and a halfo minutes, the inferior (with reference to the surgeon and not anatomically) hemicircumferential stitches of ${ }^{-}$ interrupted catgut were placed between the cut ends $\overrightarrow{\mathbb{D}}$ and tied (Fig. 4).

Once these stitches had been inserted the endo- $\stackrel{\mathbb{D}}{\circ}$ tracheal tube was left in the left main bronchus ando 


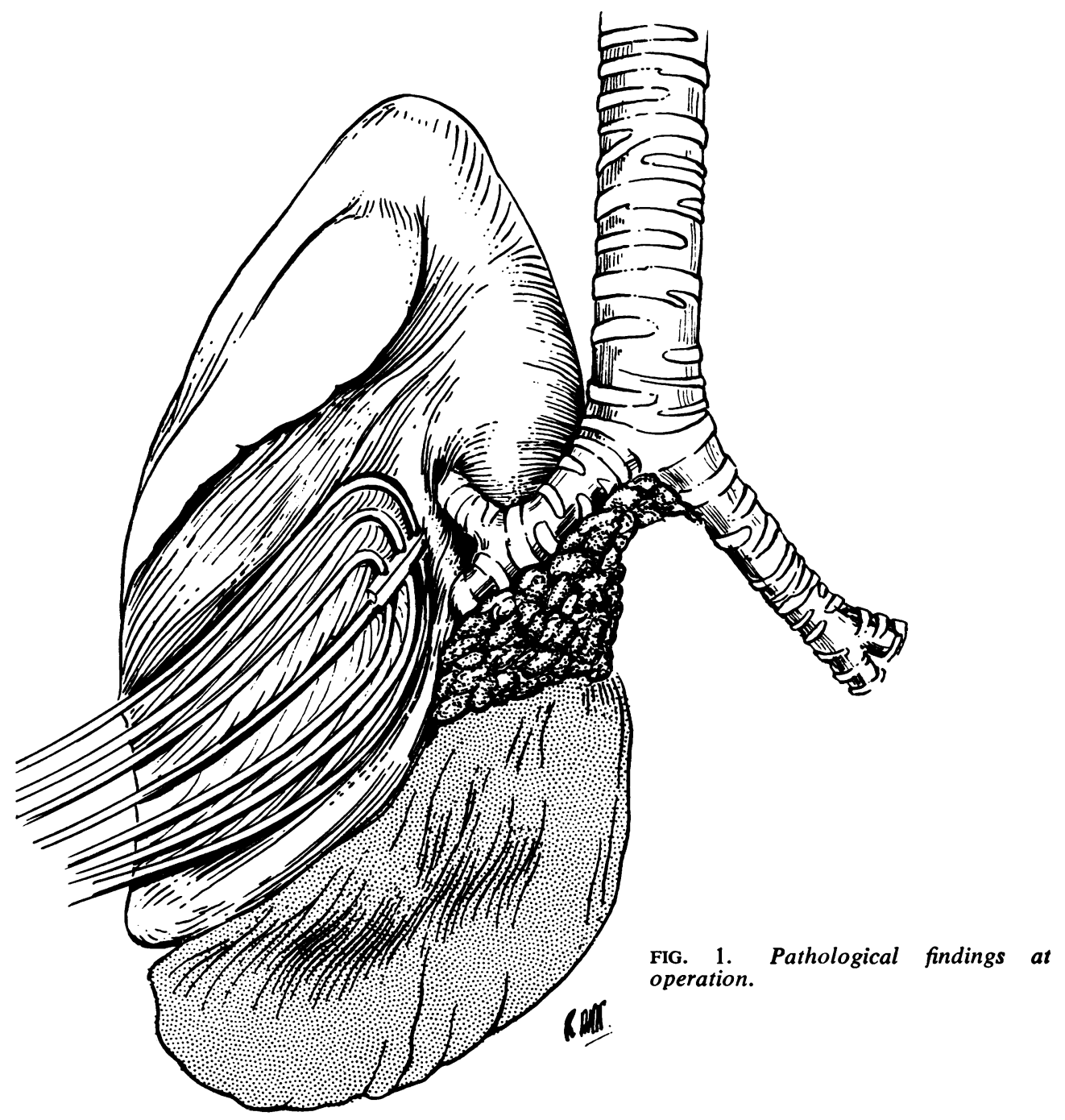

the anterior half stitches were inserted with the tube in this position (Fig. 4).

The stitches in the reconstructed trachea were the last to be inserted and tied, the final one being that inserted at the approximated cut ends.

Acriflavine was placed in the chest cavity to test for air leak on positive pressure inflation; a single small leak necessitated a further stitch.

Antibiotic powder was placed around the anastomosis, a single intercostal tube drain was inserted, and the chest was closed in layers.

The patient's post-operative course was uneventful, there was no air leak from the anastomotic site, and there were no post-operative complications such as temperature, sputum retention or the coughing up of stitches. He was discharged from hospital on the eighteenth post-operative day after an inspection bronchoscopy had shown satisfactory healing at the anastomotic site. He was readmitted to hospital three months after discharge because he was complaining of shortness of breath. Investigation, including bronchoscopy, showed no evidence of recurrence, the anastomosed lung was fully expanded, and a lung function test showed a maximum breathing capacity of $67 \mathrm{l} / \mathrm{min}$. with an F.E.V.1.0 of $86 \%$, a vital capacity of 2.21 ., and an R.V. of $45 \%$; oxygen uptake was $230 \mathrm{ml}$./min. and $\mathrm{PCO}_{2} 34 \mathrm{~mm}$. of mercury. The patient himself was a nervous man and, in view of his satisfactory lung function test, he was given chlordiazepoxide (Librium) and his dyspnoea disappeared. He was seen eight months after operation; chlordiazepoxide had been stopped, his dyspnoea had not returned, and he was capable of walking one mile in comfort at a normal speed. 

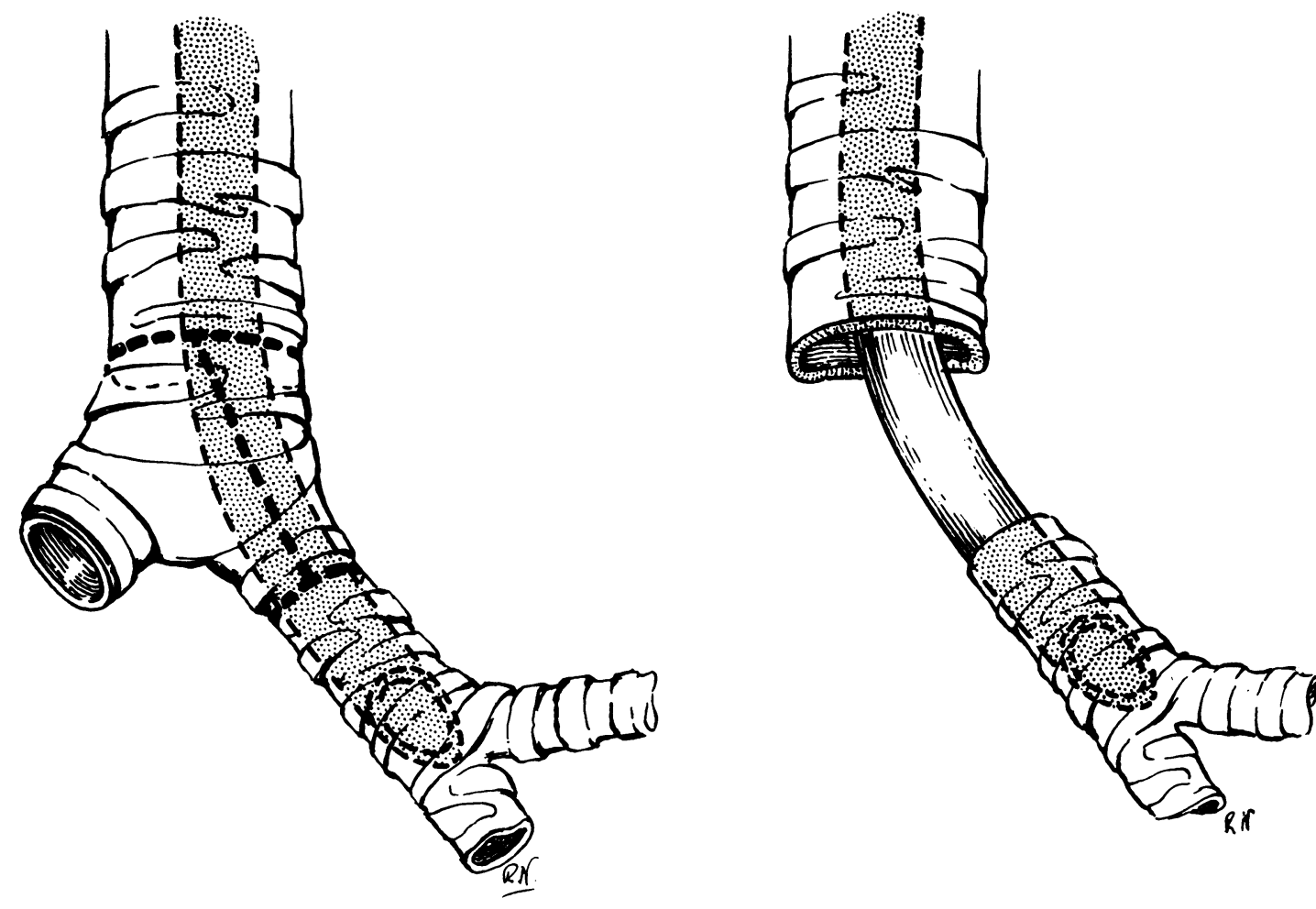

FIG. 2. Technique used for resection of carina.

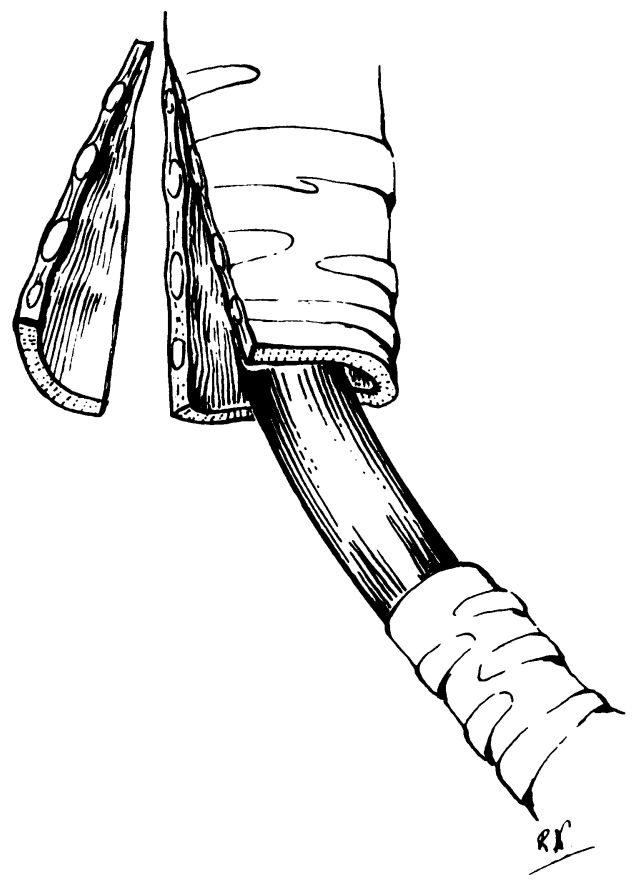

FIG. 3. Removal of wedge from trachea.

DISCUSSION

The maintenance of adequate oxygenation and gaseous exchange during the operation, while $\frac{0}{v}$ perhaps a little crude in execution, was success- $x$ fully achieved by the arbitrary decision to inflate $\frac{9}{3}$ the lung for one minute and then allow one and a half minutes for surgery. In view of the difficult $\frac{0}{3}$ situation of the anastomosis this period generally allowed one stitch to be inserted and tied. The $\mathrm{I}$ endotracheal tube was guided into the left main $\frac{D}{0}$ bronchus by the surgeon, hence trauma was minimal. In all, about six withdrawals and re- $N$ insertions of the tube were practised to allow the $N$ inferior hemi-circumferential stitches to be in- N serted.

In end-to-end tracheo-bronchial anastomoses an airtight approximation has been a stumbling block because of the difference in diameters. In $\mathbb{\Phi}$ this case an accurate anastomosis was essential because embarrassment in the function of the remaining lung could have been fatal. It was $\mathbb{\mathbb { D }}$ achieved by the excision of a triangular wedge of $\frac{?}{\mathbb{Q}}$ cartilaginous tracheal wall, leaving the anatomic- $\varrho$ ally posterior soft-tissue portion for reconstitution of the lumen. In removing this wedge, rather less? 

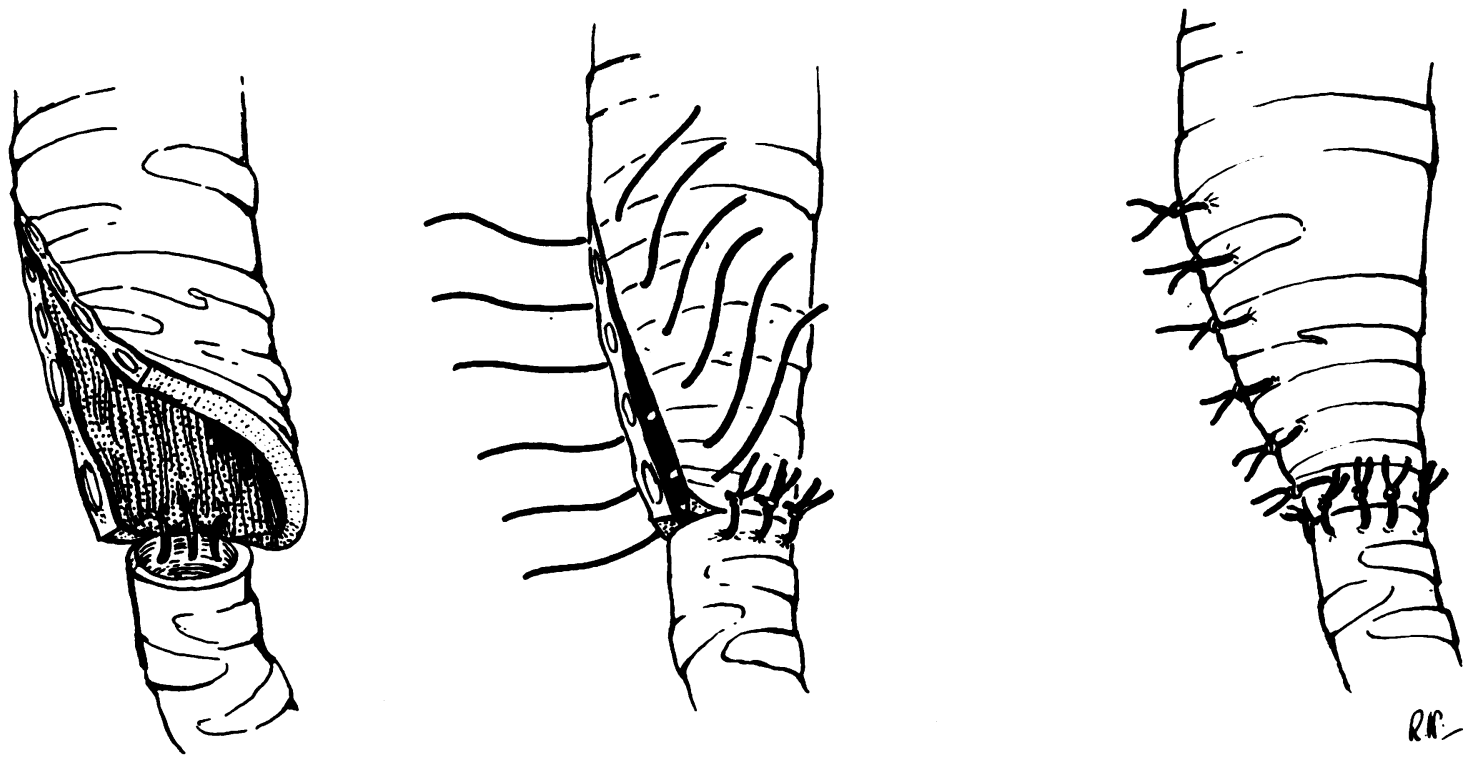

FIG. 4. Stages in execution of anastomosis (intraluminal tube omitted for clarity).

than might seem necessary was excised at first, any further reduction in lumen size could then be assessed more accurately.

Catgut stitches are recommended in bronchial anastomoses, as they are absorbed readily and hence are less likely to give rise to post-operative haemoptyses and granulation tissue, as is seen quite often in surgical procedures on the bronchus, in which non-absorbable sutures are used.

Complete expansion of the anastomosed lung was ensured immediately after the operation by aspiration bronchoscopy. During the immediate post-operative phase watch was kept on the patient's respiratory rate, and an intercostal intubation set was kept immediately available. Any sign of difficult or rapid respiration, suggesting increased compression of the anastomosed lung due to a pneumothorax, could have been confirmed immediately by insertion of a needle followed by intercostal intubation if positive intrapleural pressure had been demonstrated. As it was, no such manœuvre was necessary.

The practicability of this operation suggests that the previously accepted contraindication to resection-namely, involvement of the carina or origin of the contralateral main bronchus by tumour or involved glands-is not necessarily applicable in all cases. This procedure could also be used in ipsilateral main bronchial involvement close to the carina when normal closure techniques could not be used.
I wish to express my thanks to Mr. Richard Neave, the artist, whose diagrams have added so much to the clarity of the surgical points raised in this article; and to Dr. A. R. Hunter, the anaesthetist, for his willing and efficient cooperation.

\section{ADDENDUM}

An identical operation was performed on a patient in July 1966 without complication.

\section{BIBLIOGRAPHY}

Abbott, O. A. (1950). Experiences with the surgical resection of the human carina, tracheal wall, and contralateral bronchial wall in cases of right total pneumonectomy. J. thorac. Surg., 19, 906.

Barclay, R. S., McSwan, M., and Welsh, T. M. (1957). Tracheal reconstruction without the use of grafts. Thorax, 12, 177.

Belsey, R. (1950). Resection and reconstruction of the intrathoracic trachea. Brit.J. Surg., 38, 200.

Bryant, L. R. (1964). Replacement of tracheobronchial defects with autogenous pericardium. J. thorac. cardiovasc. Surg., 48, 733.

Gebauer, P. W. (1951). Reconstructive surgery of trachea and bronchi : late results with dermal grafts. J. thorac. Surg., 22, 568.

Grillo, H. C., Dignan, E. F., and Miura, T. (1964). Extensive resection and reconstruction of mediastinal trachea without prosthesis or graft: an anatomical study in man. $J$. thorac. cardiovasc. Surg., 48, 741.

Bendixen H. H and Gephart, T. (1963). Resection of the carina and lower trachea. Ann. Surg., 158, 889.

Macmanus, J. E., and McCormick, R. (1954). Resection and anastomosis of the intrathoracic trachea for primary neoplasms. Ann. Surg., 139, 350.

Mathey, J., Binet, J. P., Galey, J. J., Evrard, C., Lemoine, G., and Denis, B. (1966). Tracheal and tracheobronchial resections. J. thorac. cardiovasc. Surg., 51, 1.

Paulson, D. L., and Shaw, R. R. (1960). Results of bronchoplastic procedures for bronchogenic carcinoma. Ann. Surg., 151, 729.

Thomas, C. Price (1952). Carcinoma of the lung. Ann. roy. Coll. Surg. Engl., 11, 205.

(1956). Conservative resection of the bronchial tree. J. roy. Coll. Surg. Edinb., 1, 169.

Thompson, D. T. (1964). A technique for bronchial anastomosis after resection of a portion of bronchus. Thorax, 19, 347 . 